\title{
Visibility Restoration Algorithm using Bilateral Filtering
}

\author{
Seles Xavier \\ P G Student \\ Department of Computer \\ Science and Engineering \\ Federal Institute of \\ Science and Technology, Kerala
}

\author{
Reshmi R. \\ Assistant Professor \\ Department of Computer \\ Science and Engineering \\ Federal Institute of \\ Science and Technology, Kerala
}

\begin{abstract}
The visibility restoration of images is effected by various kinds of weather conditions like fog, mist, sandstorm, rain etc. This poor visibility will cause failure in the image processing applications like video surveillance, object detection, object recognition, intelligent transportation etc. The traditional visibility restoration will not requisitely restore the hazy images, because of the poor estimation of the haze thickness and color parameters. This work proposes a visibility restoration algorithm for images taken during sandstorm conditions. Haze thickness and color parameters can be accurately estimated and in turn produces a high- quality image with clear visibility and vivid color. Haze thickness estimation uses Laplacian distribution model and Gamma correction techniques. Using the estimated color parameters, each color channel can be equalized and thus overcoming the color cast problems. Finally, restoration function is used to restore the haze-free image. A bilateral filtering is proposed so as to improve the time consumption of the algorithm by replacing the soft matting technique. Thus the limitations of the traditional methods can be resolved
\end{abstract}

\section{General Terms}

Image processing, Visibility Restoration

\section{Keywords}

Gamma Correction, Dark Channel Prior, Bilateral Filter, Visibility Restoration

\section{INTRODUCTION}

Images taken during the hazy conditions like sandstorm, fog, mist etc.. contain atmospheric particles in the air. This will cause problem in the absorption and scattering between the camera and the object that is being captured (Figure.1).Thus producing a hazy image. Image visibility restoration is the technique by which the visibility of the hazy images can be restored. Image visibility restoration can be divided into three ways. They are additional information approaches, multipleimage approaches and single-image approaches. Additional information approach requires geometrical model of the captured image or the scene depth. It can be acquired by using additional operations or through user interaction. It cannot be used in real world application, since its requirement cannot practically be implemented. In multiple image approaches, more than one image of the same scene is required. It can be captured using specific hardware, in order to provide the depth information. So the cost of the additional hardware will also be included. Finally single image approaches uses only one image for de-hazing. More researches are happening in this area and it is more effective than the previous two methods.

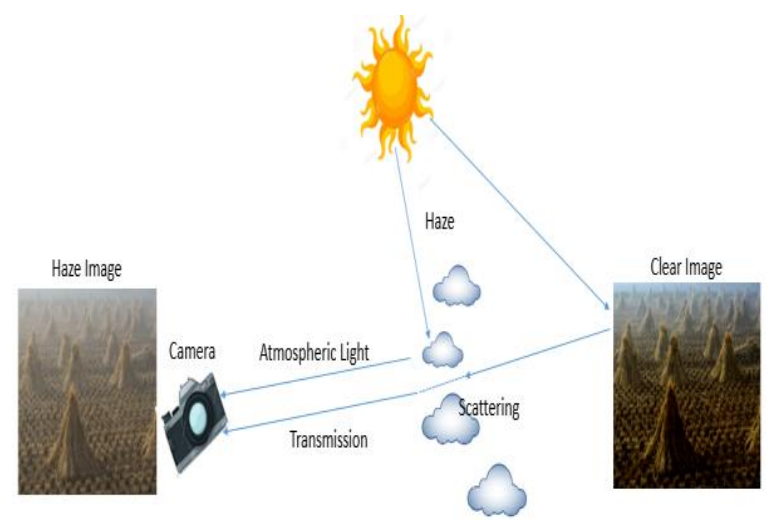

Figure.1 Haze Formation

In this work a new technique is proposed, by which visibility of the images taken during the sandstorm conditions are effectively retained. This is a single image approach. It estimates the thickness of the haze and the adjustable color parameters. Both of these are combined using a restoration function so as to effectively overcome the color cast problems and the insufficient estimation of haze thickness. Estimation of haze is based on a gamma correction technique, which refines the transmission map. Then White Patch-Retinex technique is used to effectively recover the true color scenes. Furthermore, a bilateral filter is used so as to reduce the time consumption.

The rest of the paper is organized as follows. In section II, literature survey is briefly described. Section III describes the methodology. Section IV presents the experimental results and analysis .Finally section V summarizes the system.

\section{RELATED WORKS}

Different types of visibility restoration approaches are additional information approaches (e.g. [10]), multiple-image approaches (e.g. [4]) and single-image approaches (e.g. [1],[3],[5]-[7]).Among these, single-image approaches are gaining much popularity now-a-days because of its effectiveness. Each of the following methods has their own advantages in particular areas.

In [1] Tan et al. proposed a technique based on two observations. Firstly, images taken during clear day has more contrast while comparing with images taken during the bad weather. Secondly, variation of the airlight depends on the distance between the object and the viewer. Using these assumptions cost function is developed in the framework of Markov random fields. This can be optimized using graphcuts, belief propagation etc...

In [2] Narasimhan proposed a physics based model. Instead of depth information, additional information provided by the user, along with a single image is used. This includes the 
selection of a good region in the image where colors are not corrupted. Next is the automated step. Here for each pixel, with each color in the weather affected region, search for the best color match in the good region. From this set, choose a pixel whose color is farthest from the fog color. For computing the magnitude of the replacing pixel, scene color is decomposed into two components by making use of the dichromatic model. Finally, replace the color of the pixel by the de-weathered color.

Xie et al. described a process using dark channel prior and multi-scale retinex [3].It provides automatic and fast restoration. It is based on multi scale retinex algorithm that is implemented on the luminance component in the $\mathrm{YCbCr}$ space. This way pseudo transmission map is obtained. By combing the haze imaging model and the dark channel prior, haze free image can be produced.

Narasimhan yet again presented a technique based on the polarization based approach [4]. It is a multi-image approach. It must be taken by the polarizer at different orientations. As a result, this generates a range map of the scene. This will provide the scene rendering as if it is taken from different viewpoints. As a byproduct it gets the information about the atmospheric particles.

He et al. proposed a single image haze removal based on dark channel prior. It was based on an observation that the in nonsky regions, at least one RGB color channel will have low intensity [5] .This work is based on this assumption. The image thus produced using this low intensity is called as the dark channel. Using this transmission map is obtained. Afterwards soft matting is done on the transmission map. Scene radiance is obtained by making use of a restoration function. This technique is simple and more effective for dehazing. This won't work when the objects are similar to the atmospheric light and there is no shadow.

Xie et al. presented a work using both the dark channel prior and the multi scale retinex [6].First RGB image is converted into $\mathrm{YCbCr}$ color space. This is for separating the luminance component from others. MSR algorithm is then applied on this. Median filter is applied on this to produce a pseudo transmission map. By combing the dark channel and haze imaging model, de-hazing is done. One of the main advantages is that it requires no user interaction. And the time consumption is very less.

Jin et al. proposed an algorithm based on dark channel prior [7].Here three times variance is subtracted from the mean so as to get the minimum value in a patch. This is how the dark channel image is produced. This mean and variance are calculated by the bilateral filter. This will show better results than the other methods. One of the advantages is that it does not produce halo along the edges. It can be used for real time applications. The limitation of this approach is the over estimation of the haze layer.

Gasparini et al. proposed a double step algorithm. Here the first step is the cast detection and the second is the cast removal [8]. In cast detection, it classifies into four types of cast. They are no cast, evident cast, ambiguous cast and the intrinsic casts. If there is evident or intrinsic cast then it should be removed using a cast removal. Cast removal is based on von Kries hypothesis. Von Kries co-efficient are evaluated by setting white in a particular region, called White balance region. It looks for the white balance region, which presents the low level of saturation. Thus the color is corrected. This is a quick process. But it won't correct the color in some specific areas.

Huang et al. presented a visibility restoration algorithm by combining three techniques. They are depth estimation, color analysis and the visibility restoration [9]. Depth estimation, makes use of the adaptive gamma correction and the median filter techniques. This produces transmission map. Color analysis estimates the characteristics of the colors on the input image. Visibility restoration is adjusting the transmission map and adjusting the color parameters, thus producing a haze-free image. This will eliminate the halos and produces high quality image.

\section{PROPOSED METHODOLOGY}

The proposed visibility restoration algorithm consists of three modules:

\section{- Haze Thickness Estimation}

- $\quad$ Adjustable Color Parameter Estimation

- Image Visibility Restoration

The block diagram of the overall process is given in the Figure.2,

In the haze thickens estimation module, initial transmission map was generated using the Dark Channel Prior [5].Then bilateral filtering is applied. Afterwards, the transmission map acquired after bilateral filtering is used for Gamma Correction [11], thus producing a refined transmission map. Next adjustable color parameters are found by making use of the Laplacian distribution value. Finally both the outputs of the previous two modules are used in a restoration function to produce haze-free image.

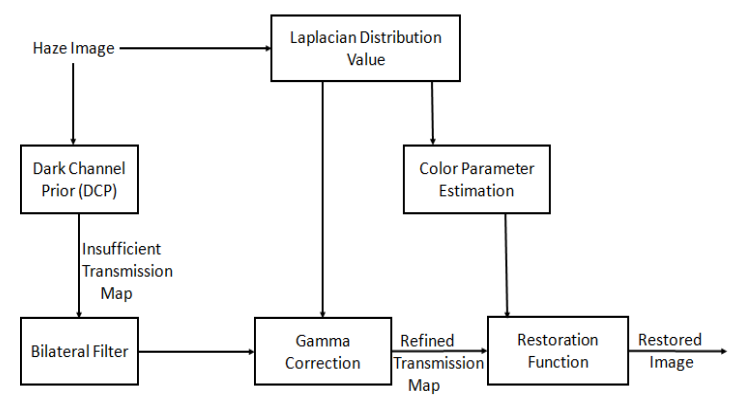

\section{Figure.2 .Block Diagram of Visibility Restoration Algorithm using Bilateral Filter}

\subsection{Haze thickness estimation}

As Haze thickness estimation was a challenging task for the dark channel prior techniques. This will produce a transmission map which is insufficient for restoring the hazy images. So here haze thickness estimation module is done using the Laplacian distribution model and the gamma correction technique. A bilateral filtering is done by replacing the traditional soft matting technique, which was time consuming. Laplacian distribution value can be calculated for each color channel in hazy image as follows,

$P\left(I^{c}\right)=\frac{1}{a} \sum_{i=0}^{a} \frac{1}{2 \sigma} e^{-\left|I^{c}(x)-\mu\right| / \sigma}$ 
where, $c \in\{r, g, b\} . I^{c}(x)$ is the incoming hazy image, a represents the number of pixels in the incoming hazy image. $\mu$ and $\sigma$ are the location and scale parameters .Location parameter is the mean of the input hazy image. Additionally, scale parameter can be defined as following,

$$
\sigma=\frac{1}{a} \sum_{i=0}^{a}\left|I^{c}(x)-\mu\right|
$$

Here, the image with slight color cast will produce high Laplacian distribution value, because of the smaller color variance. And images with high color cast have low Laplacian distribution value because of the high color variance.

Next, employ a dark channel prior technique. It is based on the observation that, some pixels has low intensity values in at least one color channel [5].The image thus created is the dark channel image. The regions with high density haze will be having high intensity in its dark channel. It can be calculated as follows,

$$
J^{\text {dark }}(y)=\min _{c \in r, g, b}\left(\min _{y \in w(x)}\left(I^{c}(x)\right)\right)
$$

A transmission map must be created next. For this each color channel of the input image is divided with corresponding atmospheric light. And the dark channel of thus produced image is taken as the transmission map. This will be insufficient .So it must be refined using the formula,

$$
t(x)=1-\omega \min _{c}\left(\min _{y \in w(x)}\left(\frac{I^{c}(x)}{A^{C}}\right)\right)
$$

where, $\omega$ is set to $0.95 . I^{c}(x)$ is the input hazy image, $A^{c}$ is the atmospheric light estimation for each color channel of the input hazy image.

After finding the dark channel prior, fast bilateral filtering is done. It is done instead of the time consuming soft matting that is being done in the traditional methods. A bilateral filter is a noise reducing smoothing as well as edge preserving filter. Here the intensity value of each pixel is being replaced by a weighted average of the intensity values of the neighbor pixels. This weight can be based on a Gaussian distribution. This will preserve the sharp edges by looping each pixel and then adjusting the weight of the neighbor pixels. This can be achieved by making use a filter. Next gamma correction is employed with self adaptive parameters. This is used to refine the transmission map that is acquired using fast bilateral filter. Gamma value is obtained by making use of the Laplacian distribution value. It is defined as follows,

$$
\gamma=\frac{\max _{c}\left(P\left(I^{c}\right)\right)}{\min _{c}\left(P\left(I^{c}\right)\right)}
$$

where, max and min represents the maximum and minimum value between the RGB color channels. Higher values of selfadaptive parameters indicate higher refinement efficacy. The insufficient transmission map can be refined using the gamma obtained. It can be defined as follows,

$$
t(x)=l_{\max } *\left(\frac{\tilde{t}^{\gamma}}{l_{\max }}\right)^{\gamma}
$$

where, $l_{\max }$ represents the maximum value of a pixel and can be set to 1. $\tilde{t}(x)$ denotes an insufficient transmission map, which is acquired using bilateral filtering.

\subsection{Adjustable color parameter estimation}

In the previous section, Laplacian distribution values and refined transmission map are generated. Next the color parameters that must be adjusted should be estimated. Because of the insufficient amounts of color variation, there will be color cast problems in the input image. So in order to avoid such problems employ a Laplacan based White Patch Retinex technique to recover the true color scenes. Adjustable color parameters can be found as follows,

$$
\alpha^{c}=\frac{v^{c}}{\max \left(P\left(I^{c}\right)\right)}
$$

where,

$$
v^{c}=\frac{\max \left(P\left(I^{c}\right)\right)}{P\left(I^{c}\right)}
$$

$c \in\{r, g, b\}, \max _{c} I^{c}$ represents the maximum intensity of each color channel in the input hazy image. $v^{c}$ are the chromatic adaptable parameters, max represents the maximum values between the color channels. Inconsistent distributions of the color channel in the hazy image are represented using higher values in the chromatic adaptable parameter.

\subsection{Image Visibility Restoration}

High quality haze free image with vivid color and clear visibility can be generated in this module. This is achieved by using the outputs of previous modules. That is the output of haze thickness estimation is refined transmission map and the next module produced adjustable color parameters. By using these two values in a restoration equation visibility of the hazy image can be restored. The restoration function is as follows,

$$
J^{c}=\frac{\alpha^{c}\left(I^{c}(x)-A^{c}\right)}{\max \left(t(x), t_{0}\right)}+\alpha^{c} A^{c}
$$

where , $c \in\{r, g, b\}, \quad J^{c}(x)$ represents the scene radiance, $I^{c}(x)$ is the hazy image captured by the digital camera. $A^{c}$ is the global atmospheric light, $\alpha^{c}$ represents the adjustable color parameters and $t_{0}$ is set to 0.1.By using this restoration function the visibility of the hazy input image can be restored effectively.

\section{EXPERIMENTAL RESULTS AND ANALYSIS}

This visibility restoration algorithm using bilateral filtering was implemented using MATLAB 2009 on Intel i3, 1.70GHz processor with 4GB RAM, running on Windows 8 operating system. The data base was built with images from Google, Flicker etc...The dataset contained different kinds of weather conditions. The following Table.1 shows the values that have been set to the variables throughout this work.

Table.1 Values Assigned

\begin{tabular}{|c|c|c|}
\hline Sl. No. & Variable & Value \\
\hline 1 & Patch Size & $15 \times 15$ \\
\hline
\end{tabular}




\begin{tabular}{|c|c|c|}
\hline 2 & Pad Size & $7 \times 7$ \\
\hline 3 & $\Omega$ & 0.95 \\
\hline 4 & $\Lambda$ & $10^{-2}$ \\
\hline 5 & sigmas & 4 \\
\hline 6 & sigmar & 50 \\
\hline 7 & tol & 0.1 \\
\hline 8 & Window Size & 50 \\
\hline 9 & $\mathrm{t}_{0}$ & 0.1 \\
\hline
\end{tabular}

The input hazy image for the proposed method is shown in Figure.3,

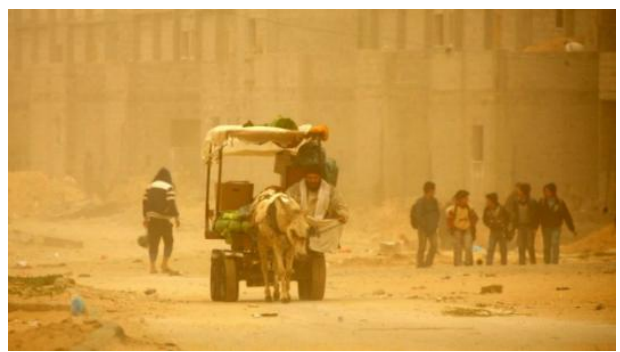

Figure.3. Input hazy image

Next the Dark channel image of this can be seen inFigure.4,

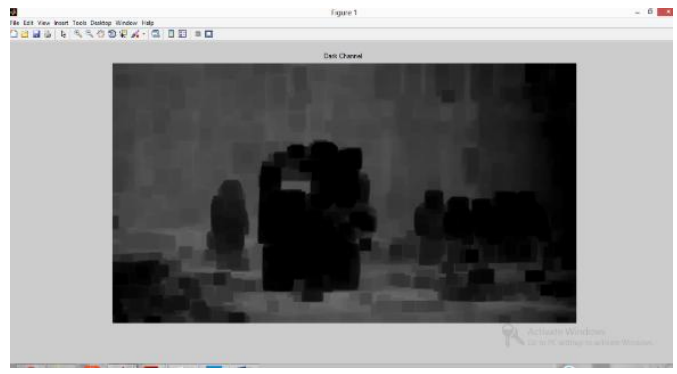

Figure.4. Dark Channel Image

After that the insufficient transmission map is obtained as in Figure.5,

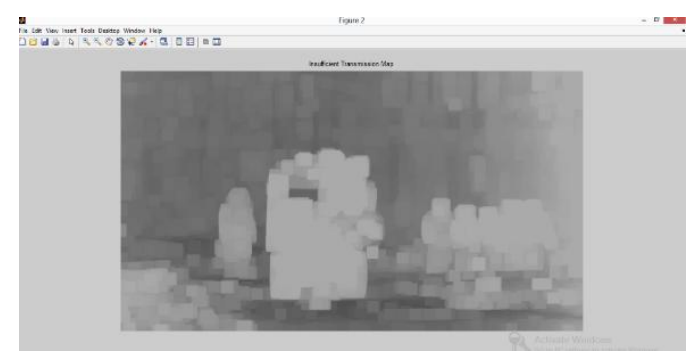

Figure.5.Insufficinet Transmission Map

Next, bilateral filtering is done and the transmission map acquired after it is given in Figure.6,

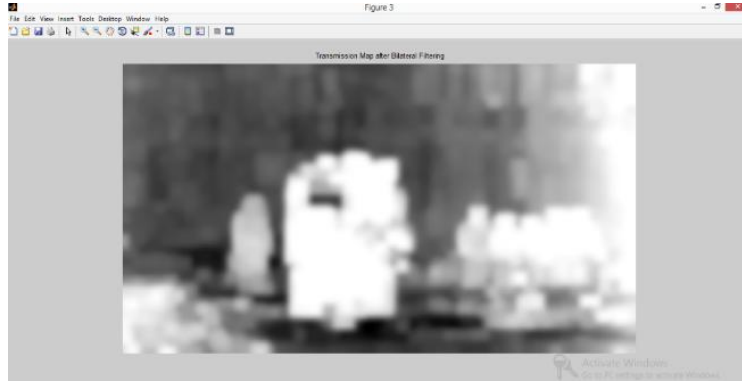

Figure.6.Transmission map acquired after bilateral filtering

Finally the transmission map is refined using the gamma correction technique in Figure.7,

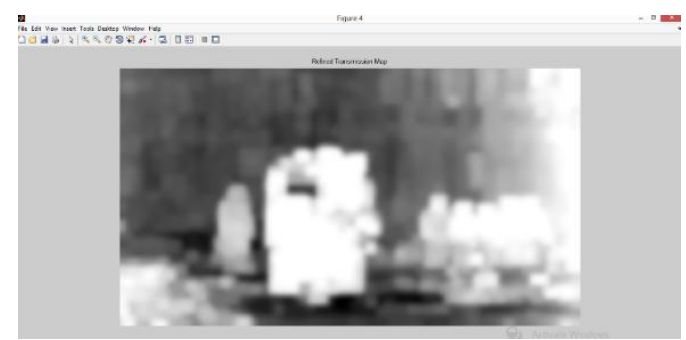

Figure.7.Refined Transmission Map

After computing the adjustable color parameters, a restoration function is used to restore the visibility of the degraded images. The finally restored image is given in Figure.8,

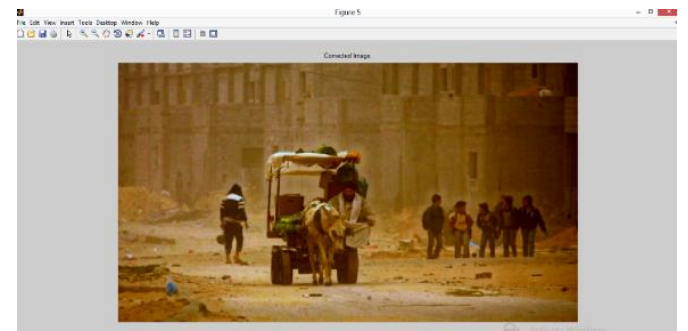

Figure.8.Restored image

Next some of the hazy images and their restored output images can be seen in Figures.9, 10, 11, 12.

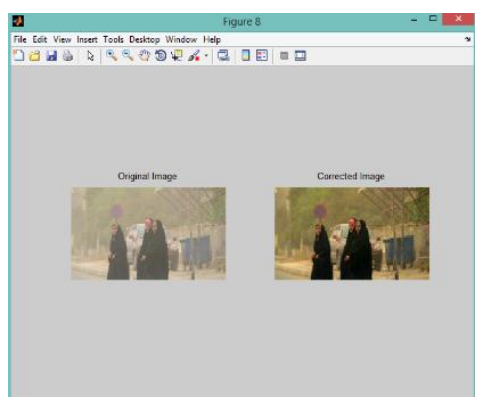

Figure.9. Original image (left) and restored image (right) of Pedestrian Image 


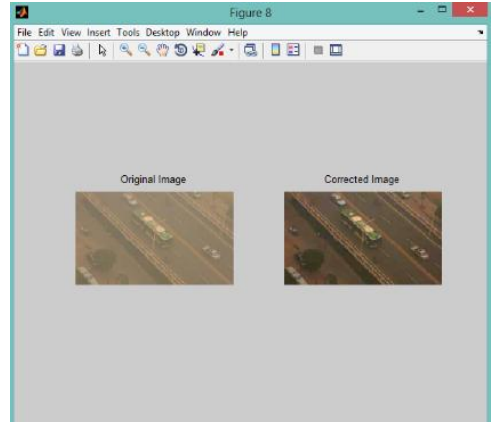

Figure.10 Original image (left) and restored image (right) of Highway Image

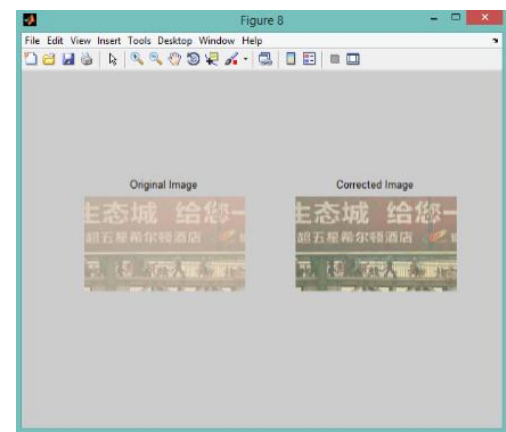

Figure.11 Original image (left) and restored image (right) of Signboard Image

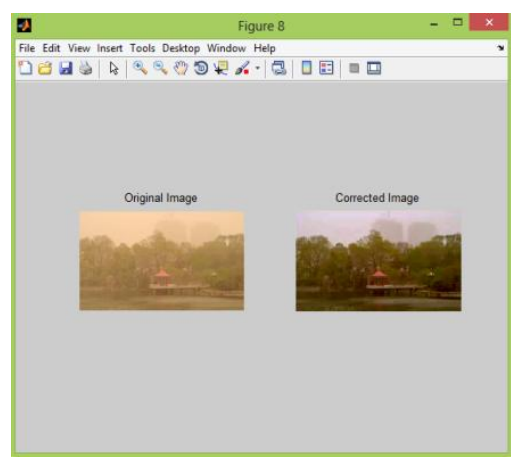

Figure.12. Original image (left) and restored image (right) of Park Image

In order to validate this method, the results obtained by implanting this are compared with that of the existing methods. For analyzing the performance of the proposed method the values of Relative Contrast Error, Relative Structural Similarity, Relative Entropy Error and Relative Mean Brightness Errors, Peak Signal to Noise Ratio and Mean Square Error are calculated between the original image and the restored image for the test images in Table.6.

The value of REE is more than 0.5 , which means that the image data is increased. If it is less than 0.5 , this means that the image is deteriorated. While $\mathrm{RMBE}=1$, it indicates that the mean brightness of the restored image remains unchanged. But when it is equal to zero, it means that there is greater difference in the mean brightness between the input hazy image and the output haze -free image. RSS is to define the degree of structural similarity between the original image and the output image. If there is greater structural similarity, then RSS will be close to 1 and greater structural difference shows RSS $=0$. PSNR used as the quality measurement between the original and restored images [12].Greater PSNR value is
good.MSE is the cumulative squared error between the restored and original image. Lower MSE value is good [12].

$\omega$ can be varied and the performance can be evaluated. It can be varied from 0.10 to2.0.The values at taken at random. From the Table.2, 3, it is clear that the images with lesser $\omega$ value will have good PSNR and MSE values. But the brightness in these images is more.REE is almost same for all. While giving higher values like 1.5 for $\omega$, the PSER and MSE values are getting worse and the image is almost black. So for this work a middle value is taken. So the PSNR and MSE are comparable and the image is better than he images at the two extreme values.

Table 2. Varying $\omega$ values

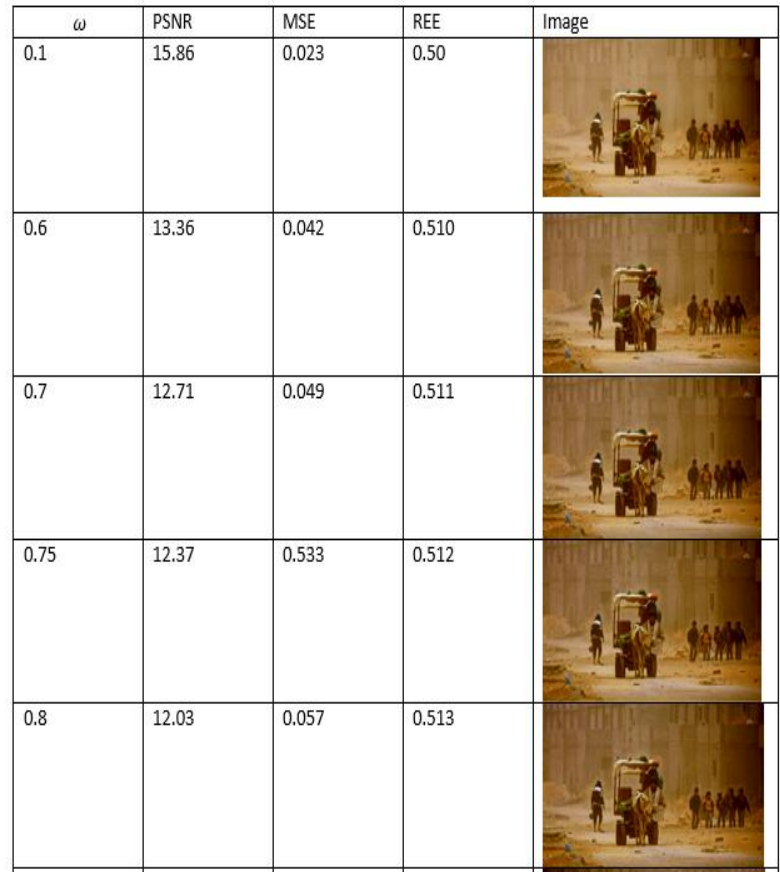

Table.3. Varying $\omega$ value

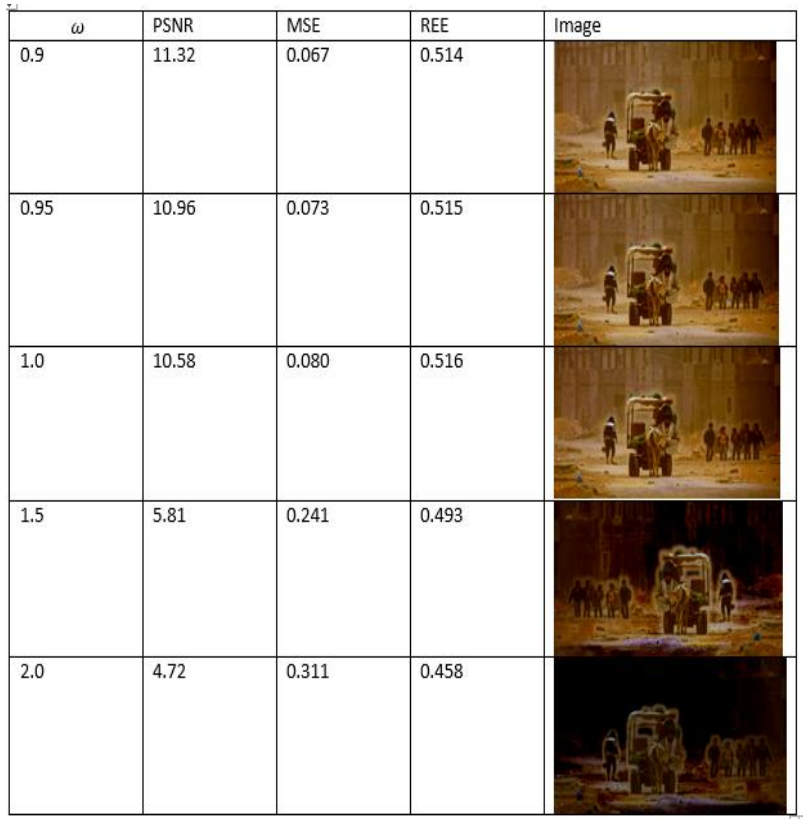


Table.4.Varying Pad Size

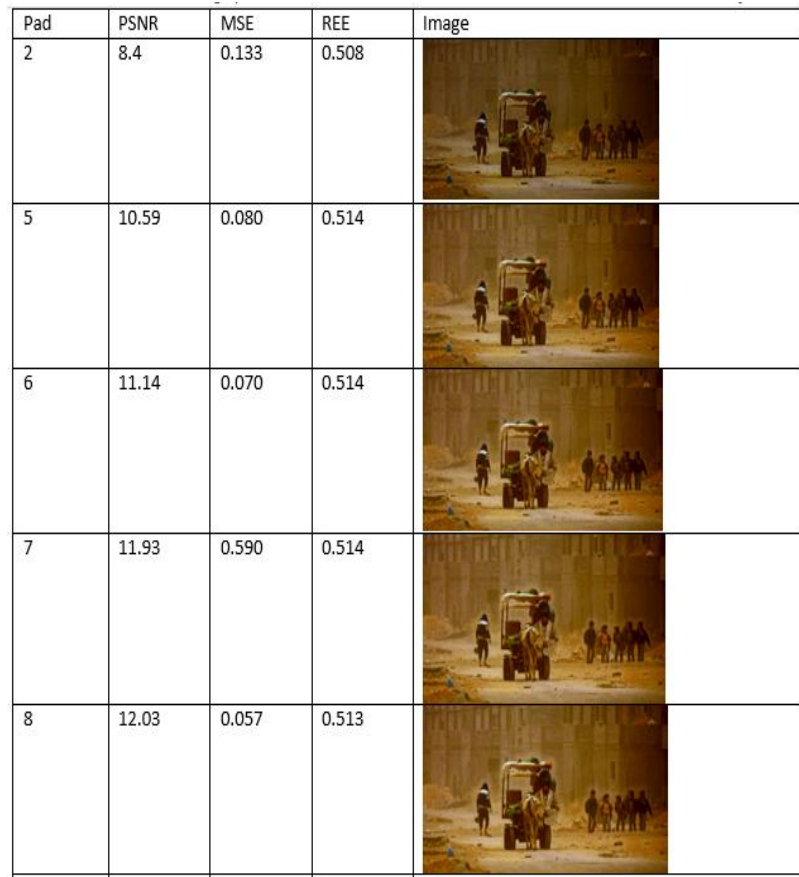

Next, the pad size can be varies and the performance can be evaluated. From the Table.4, 5, it is clear that as the pad size increases there are distortions in the image. A white smoke is appearing at the edge as the size increases. Even if PSER shows better results as the pad size increases, the image is getting distorted. MSE and REE vales are comparable. From these observations it is better to choose a middle value instead of these two extremes.

\section{Table 5.Varying Pad Size}

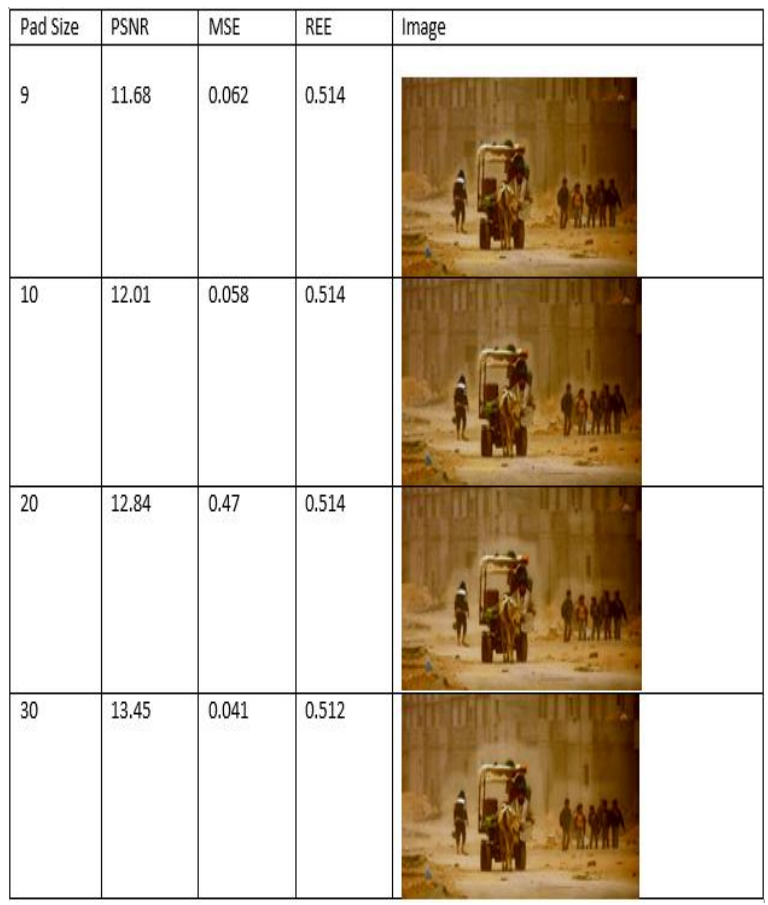

Analysis of the existing method [12] and the proposed method are given in the Table.6 .Here values obtained for RCE, RMBE, RSS are having same values for both the implementations. The RCE value is 0.50 for all the methods. This indicates that the contrast of the images has not been enhanced .RMBE is 1.0 for both the methods. This indicates that the mean brightness of the restored image is remaining unchanged. Next is the RSS. It is 1.0 for all the images. This shows higher structural similarity. REE gives comparable results for all. For Fig.9, in the existing method it gives 0.4975 which is less than 0.5 and in the proposed method it is 0.5159 , which is greater than 0.5.So it shows good results. PSNR value is much greater for proposed method. Higher the PSNR is the better. Next MSE is less for the proposed method. Lesser the MSE is the best. So it also satisfies our method.

Table.6.Analysis of Existing Method and the proposed Method using REE, RCE, RMBE, RSS, PSNR, MSE

\begin{tabular}{|c|c|c|c|c|c|c|c|}
\hline $\begin{array}{l}\text { Algorit } \\
\mathrm{hm}\end{array}$ & $\begin{array}{l}\text { Figu } \\
\text { re }\end{array}$ & REE & $\begin{array}{l}\text { RC } \\
\mathrm{E}\end{array}$ & $\begin{array}{l}\text { RM } \\
\text { BE }\end{array}$ & $\begin{array}{l}\text { RS } \\
\text { S }\end{array}$ & $\begin{array}{l}\text { PSN } \\
\text { R }\end{array}$ & $\begin{array}{l}\text { MSR } \\
\text { E }\end{array}$ \\
\hline \multirow{5}{*}{$\begin{array}{l}\text { Existin } \\
\text { g } \\
\text { System }\end{array}$} & $\begin{array}{l}\text { Fig. } \\
8\end{array}$ & $\begin{array}{l}0.51 \\
20\end{array}$ & 0.5 & 1.0 & 1.0 & 6.17 & 0.24 \\
\hline & $\begin{array}{l}\text { Fig. } \\
9\end{array}$ & $\begin{array}{l}0.49 \\
75\end{array}$ & 0.5 & 1.0 & 1.0 & $\begin{array}{l}10.6 \\
1\end{array}$ & 0.77 \\
\hline & $\begin{array}{l}\text { Fig. } \\
10\end{array}$ & $\begin{array}{l}0.52 \\
10\end{array}$ & 0.5 & 1.0 & 1.0 & 8.34 & 0.09 \\
\hline & $\begin{array}{l}\text { Fig. } \\
11\end{array}$ & $\begin{array}{l}0.52 \\
24\end{array}$ & 0.5 & 1.0 & 1.0 & 7.91 & 0.13 \\
\hline & $\begin{array}{l}\text { Fig. } \\
12\end{array}$ & $\begin{array}{l}0.50 \\
57\end{array}$ & 0.5 & 1.0 & 1.0 & $\begin{array}{l}11.1 \\
8\end{array}$ & 0.57 \\
\hline \multirow{5}{*}{$\begin{array}{l}\text { Propose } \\
\text { d } \\
\text { System }\end{array}$} & $\begin{array}{l}\text { Fig. } \\
8\end{array}$ & $\begin{array}{l}0.51 \\
12\end{array}$ & 0.5 & 1.0 & 1.0 & $\begin{array}{l}10.8 \\
8\end{array}$ & 0.08 \\
\hline & $\begin{array}{l}\text { Fig. } \\
9\end{array}$ & $\begin{array}{l}0.51 \\
59\end{array}$ & 0.5 & 1.0 & 1.0 & $\begin{array}{l}13.2 \\
6\end{array}$ & 0.04 \\
\hline & $\begin{array}{l}\text { Fig. } \\
10\end{array}$ & $\begin{array}{l}0.51 \\
84\end{array}$ & 0.5 & 1.0 & 1.0 & $\begin{array}{l}12.2 \\
4\end{array}$ & 0.03 \\
\hline & $\begin{array}{l}\text { Fig. } \\
11\end{array}$ & $\begin{array}{l}0.51 \\
51\end{array}$ & 0.5 & 1.0 & 1.0 & $\begin{array}{l}16.2 \\
9\end{array}$ & 0.02 \\
\hline & $\begin{array}{l}\text { Fig. } \\
12\end{array}$ & $\begin{array}{l}0.51 \\
06\end{array}$ & 0.5 & 1.0 & 1.0 & $\begin{array}{l}11.7 \\
1\end{array}$ & 0.05 \\
\hline
\end{tabular}

Next is the processing time. The proposed method that makes use of the bilateral filter is takes much less time while comparing with the existing methods. It is one of the main advantages of using this technique. It can be clearly seen from the Table.7

So, in short the proposed method is giving better PSNR, MSE and REE values. And RCR, RMBE and RSS are giving comparable results. The time consumption is much reduced in the proposed method. So from these, it is clear that the proposed visibility restoration technique using bilateral filtering is much better than the state-of art methods.

Table.7.Processing Time of Existing Method and the Proposed Method

\begin{tabular}{|l|l|l|}
\hline Algorithm & Figure & Time(s) \\
\hline $\begin{array}{l}\text { Existing } \\
\text { System(Using } \\
\text { Soft Matting) }\end{array}$ & Fig.8(162 x 121) & 153.644 \\
\cline { 2 - 3 } & Fig.9(162 x 96) & 116.400 \\
\cline { 2 - 3 } & Fig.10(161 x 96) & 117.347 \\
\cline { 2 - 3 } & Fig.11(163 x 96) & 119.480 \\
\hline
\end{tabular}




\begin{tabular}{|c|c|c|}
\hline & Fig.12(98 x 163) & 123.08 \\
\hline \multirow{5}{*}{$\begin{array}{l}\text { Proposed } \\
\text { System(Using } \\
\text { Bilateral Filter ) }\end{array}$} & Fig.8(162 x 121) & 08.832 \\
\hline & Fig.9(162 x 96) & 10.791 \\
\hline & Fig.10(161 x 96) & 10.358 \\
\hline & Fig.11(163 x 96) & 10.562 \\
\hline & Fig.12(98 x 163) & 12.24 \\
\hline
\end{tabular}

\section{CONCLUSION}

The work proposes a visibility restoration algorithm using bilateral filtering for the sandstorm images. This makes use of the haze thickness and adjustable color parameters to restore the visibility of the hazy images. Haze thickness estimation is done by making use the Dark Channel Prior and the Gamma correction techniques. Whereas, the color parameter estimation is done using the White Patch Retinex theory. Finally using a restoration function, the visibility of the images can be restored. The experimental results show that the proposed work is superior to that of the existing methods. This work has more PSNR and MSE values when compared with other methods. The proposed work using the bilateral filtering has less time consumption while compared with the other state-of-art methods.

\section{REFERENCES}

[1] R. Tan.2008. Visibility in bad weather from a single image. iProc. IEEE Conf. CVPR.

[2] S. G. Narasimhan and S. K. Nayar.2003.Interactive (De)weathering of an image using physical models. Proc. ICCV Workshop Color Photometr. Methods Comput. Vis.
[3] B. Xie, F. Guo, and Z. Cai.2010. Improved single image dehazing using dark channel prior and multi-scale Retinex. Proc. Int. Conf. Intell. Syst. Des. Eng. Appl.

[4] Y. Y. Schechner, S. G. Narasimhan, and S. K. Nayar.2003. Polarization based vision through haze.Appl. Opt.

[5] K. He, J. Sun, and X. Tang. 2011. Single image haze removal using dark channel prior. IEEE Trans. Pattern Anal. Mach. Intell.

[6] B. Xie, F. Guo, and Z. Cai.2010. mproved single image dehazing using dark channel prior and multi-scale

[7] F. Gasparini and R. Schettini. 2003. Color correction for digital photographs .Proc. IEEE Int. Conf. Image Anal. Process.

[8] S. C. Huang, B. H. Chen, and W. J.Wang. 2014. Visibility restoration of single hazy images captured in real-world weather conditions. IEEE Trans. Circuits Syst. Video Technology.

[9] K. Tan and J. P. Oakley.2000.Enhancement of color images in poor visibility conditions. Proc. IEEE ICIP.

[10] S. C. Huang, F. C. Cheng, and Y. S. Chiu.2013.Efficient contrast enhancement using adaptive gamma correction with weighting distribution, IEEE Trans. Image Process.

[11] Shih-Chia Huang ,Jian-Hui Ye ,Bo-Hao Chen.An Advanced Single-Image Visibility Restoration Algorithm for Real-World Hazy Scenes. IEEE Transactions on Industrial Electronics. 